\title{
EDITORIAL
}

\section{The surgeon should assume a prominent role in the diagnosis and treatment of gastro-oesophageal reflux disease}

A few months ago, in February, I had the privilege of participating in a panel discussion in front of members of the College of Surgeons of Sri Lanka as well as pathologists where questions regarding the diagnosis and management of gastro-oesophageal reflux disease (GORD) were discussed. It was quite apparent from the questions presented, that a great deal of thought and insight existed on the part of College members about the important issues in caring for the GORD patient. Central to the discussion was the question, "What is the role of the general surgeon in the management of this disease?"

In the United States, we are taught that GORD is a disease of the western world, primarily seen in the US and Europe due to diet, the epidemic of obesity and other factors. It is the most common gastrointestinal disorder seen by both primary care physicians and gastroenterologists. We are also taught that reflux disease is rarely seen in Asia and other eastern countries such as Sri Lanka. However, it became quite apparent that GORD is seen with increasing frequency in Sri Lanka and the same problematic questions regarding efficient diagnosis and management that we struggle with in the US, also exist in this nation.

The evolution of GORD management in the United States has unfortunately led to an undesirable "status quo". A patient presents, usually to the primary care physician with symptoms such as heartburn, regurgitation or any other foregut complaint. The physician concludes that the symptom source is possibly reflux disease and the patient is placed on empiric proton pump inhibitor therapy (PPIs) and sent on his way. If symptoms resolve, patients are left on their PPIs, often indefinitely. Persistent symptoms are often further investigated by a gastroenterologist with an endoscopy, which usually is without significant findings. Patients are typically continued on PPIs and returned to the care of the primary care physician without resolution of their problem. This method of management has led to runaway PPI costs, exceeding 10 billion dollars annually. We have continued this approach and watched the incidence of reflux-induced oesophageal adenocarcinoma increase more then $600 \%$ in the past 40 years [1] and millions continue to suffer under medical therapy often augmenting their PPIs with antacids and $\mathrm{H} 2$ blockers without relief. The present approach is failing and based on the medical literature, that should be of no surprise.

Making the diagnosis of GORD based on patient symptoms alone is fraught with hazard. Some $30-40 \%$ of patients, including those presenting with typical symptoms of heartburn and regurgitation, are found not to have reflux disease after undergoing $\mathrm{pH}$ testing. Furthermore, while upper endoscopy is necessary to rule out other disorders, it is a terrible test for the presence or absence of GORD with objective findings of reflux (erosive oesophagitis, stricture or Barrett's oesophagus) present in only $20 \%$ of patients [2]. Symptomatic response to PPI therapy also incorrectly predicts the presence or absence of reflux disease in as many as $50 \%$ of patients [3]. Yet these are the "tools" typically used in the diagnosis of reflux that have led to the unsatisfactory management we observe today. The availability in recent years of over the counter PPIs, allowing patients to self medicate has made this problem even greater.

From a therapeutic standpoint, more problems are seen. Upon their introduction, PPIs were thought to be the panacea due to their powerful acid suppressive effects. However, we now understand that approximately $40 \%$ of GORD patients continue to have breakthrough symptoms on PPIs [4]. Continued symptomatic complaints are frequently ignored by medical physicians or addressed by escalating the dose of these medications, often without benefit. The immense experience with PPIs over the years, has unmasked disturbing side effects. These include an increased risk of hip fractures and increased risk of C. difficile colitis, both of which have prompted Food and Drug Administration alerts in the US. Hypomagnesemia, increases in community acquired pneumonias and other side effects are of concern. Many patients are also never told that this medical therapy is usually necessary for life and other options such as surgery are never discussed or offered. One must question the desirability of a therapy that requires daily medications for life, is extremely costly, has significant side effects and resolves a patient's symptoms only about $60 \%$ of the time. Certainly, 
specific efforts in developing an approach where other therapeutic choices, including surgery, could provide great patient benefit.

The endemic use of PPIs may also be playing a role in the dramatic and disturbing rise in the incidence adenocarcinoma of the oesophagus. Even the American Gastroenterological Association in its 2011 Guidelines on the management of Barrett's oesophagus acknowledged the possibility that PPI-induced gastric $\mathrm{pH}$ changes could possibly be adversely altering the chemistry of the refluxate and contribute to the rise in reflux-induced cancer [5]. This should be an alarming concept, yet it is infrequently discussed and has not significantly affected PPI use. In that same publication, the possibility that anti-reflux surgery may protect against development of cancer by stopping the reflux was also discussed.

Despite the above considerations, the present empiric medical approach seems entrenched in the United States and significant change will be very difficult. However, as more and more GORD is seen in Sri Lanka, a different course, recognizing these problematic issues could be taken. Involvement of the surgeon in both the diagnostic and therapeutic aspects of GORD would allow a more effective approach to this disease that would greatly benefit patients. The surgeon, however, must position himself not as an individual interested in only performing an operation, but as someone with a true understanding of reflux and oesophageal disease, capable of proper diagnostic evaluations, patient education and familiarity with all potential therapies. Only the surgeon can offer all options and tailor such offerings to each specific patient.

Anti-reflux surgery has been proven to be an effective long-term therapy for GORD [6]. In expert hands, it provides symptomatic relief, eliminates the need for acid suppressive medications and may decrease the likelihood of a patient developing of oesophageal adenocarcinoma. It is the only approach that actually stops the reflux and this had been confirmed with postoperative $\mathrm{pH}$ study normalization in $80-90 \%$ of patients. Although there are known side effects, these are minimal in the majority of patients if a proper pre-operative evaluation is performed. Persistent dysphagia should be seen in no more than 2-5\%. Surgery tends to be offered to the most severely symptomatic patients and even in this group, satisfaction is high with symptom resolution in over $80 \%$ [7]. This exceeds efficacy and satisfaction with the entire group of PPI patients, many of whom have relatively minimal symptoms. Conceptually, the surgical approach makes sense as it directly addresses the cause of the disease by re-establishing the integrity of the lower oesophageal sphincter (LOS) and stopping the reflux. Medical therapy with PPIs does absolutely nothing for the LOS and the reflux is not reduced. Only the composition of the refluxate is changed and for many patients this is not sufficient. As noted above, it could possibly be quite harmful.

The efficacy of laparoscopic Nissen fundoplication has been proven and the concept of a therapy that directly restores the function of the LOS has prompted the development of a host of endoscopic approaches over recent years. Most have come and gone due to lack of efficacy. The Stretta procedure and transoral intraluminal fundoplication (TIF) remain available, but their efficacy is debated and neither has demonstrated predictable improvement in distal oesophageal acid exposure. They do not predictably decrease reflux and therefore their efficacy in positively influencing a dysfunctional LOS is questioned.

LINX (Torax Medical, Minneapolis, MN, USA) is a relatively new procedure that has been available for several years. A small ring of titanium magnetic beads is placed around the LOS using a laparoscopic procedure that takes approximately 30 minutes and requires minimal dissection. The procedure can be done as an outpatient. The ring does not compress the LOS. It is placed loosely and "augments" the LOS. The beads separate when a food bolus is passed into the stomach and then the magnets return to their resting state providing augmentation to the damaged LOS thereby preventing reflux. Over 3000 LINX procedures have been performed worldwide and follow up of greater than 5 years is available. LINX provides predictable symptom resolution in $>80 \%$ without the side effects seen with Nissen fundoplication. Normalization of $\mathrm{pH}$ is seen in the majority of patients confirming the desired result [8]. Sphincter augmentation with LINX results in restoration of the barrier to reflux. Medications can usually be completely discontinued. Exclusion criteria include hiatus hernia $>3 \mathrm{~cm}$. 
Only the surgeon can offer all of the treatment options. To do so effectively, he must understand and apply knowledge of all aspects of GORD. This includes a meticulous evaluation of patients and incorporation of $\mathrm{pH}$ testing and high resolution oesophageal manometry. Understanding the utilization and interpretation of these diagnostics are essential in the logical management of GORD. This requires quite a commitment on the part of the surgeon but will translate into optimal patient care with proper utilization of both medical and surgical therapy.

To achieve this end, I propose the establishment of a "Reflux Center" for the evaluation and treatment of GORD and oesophageal disease. This entity should be overseen by the general surgeon or collaboratively with gastroenterologists who want to advance beyond the "scope and a pill, one size fits all" approach. Symptomatic patients would undergo complete evaluation including endoscopy, biopsies, $\mathrm{pH}$ testing and high resolution manometry, providing an objective diagnosis for each. For many, GORD will be ruled out. Some will be found to have a primary oesophageal motility disorder such as achalasia or nutcracker oesophagus. GORD patients failing medical therapy or desiring an alternative will be educated and offered a surgical approach.

The incidence of GORD in Sri Lanka is likely to increase and for the benefit of your patients a logical, programmatic approach is encouraged with the general surgeon taking a lead role.

\section{William C. Dengler, MD, FACS}

wdengler@legatomedical.com

\section{References:}

1. Chandrasoma PT, DeMeester TR. GERD: From Reflux to Adenocarcinoma. $1^{\text {st }}$ ed. Burlington MA, USA: Academic Press 2006

2. Richter JE, Pandolfino JE, Vela MF, Kahrials PJ, Lacy BE, Ganz R, Dengler W, Oelschlager BK, Peters J, DeVault KR, Fass R,GyawaliCP, Conklin J, DeMeester T. Utilization of wireless $\mathrm{pH}$ monitoring technologies:a summary of the proceedingsfrom the Esophageal Diagnostic Working Group. Dis Esophagus 2013;26(8):755-765.

3. Bytzer P, Jones R, Vakil N, Junghard O, Lind T, Wernersson B, Dent J. Limited ability of the proton-pump inhibitor test to identify patients with gastroesophagealreflux disease. ClinGastroenterolHepatol 2012;10 (12):1360-1366.

4. AGA Institute: GERD patient study: patients and their medications. Harris Interactive 2008

5. Spechler SJ, Souza RF, Inadomi JA, Shaheen N, Allen JI, Brill J, Pruitt RE, Kahrilas PJ, Peters J, Nix K, Montgomery EA, MitchellDB, Yao J. American Gastroenterological Association medical position statement and technical review on the management of Barrett's Esophagus. Gastro 2011; 140 (3): 1084-1091.

6. Katz PO, Gerson LB, Vela MF. Guide lines for the diagnosis and management of gastroesophagealreflux disease. Am J Gastroenterol 2013; 108 (3):308-28.

7. Oelschlager BK, Ma KC, Soares RV, Montenovo MI, Munoz Oca JE, Pellegrini CA. A broad assessment of clinical outcomes after laparoscopic anti-reflux surgery. Ann Surg 2012;256 (1):87-94.

8. Bonavina L, Saino G, Bona D, Sironi A, Lazzari V. One hundred consecutive patients treated with magnetic sphincter augmentation for gastroesophageal reflux disease: 6 years of clinical experience from a single center. J AmColl Surg 2013;217(4): 577-85. 